\title{
Ictal involvement of the nigrostriatal system in subtle seizures of ring chromosome 20 epilepsy
}

\author{
*Stefano Meletti, †Aglaia Vignoli, *Francesca Benuzzi, $\$$ Pietro Avanzini, *Andrea Ruggieri, \\ *Matteo Pugnaghi, *Paolo Nichelli, and $\dagger \oint$ Maria Paola Canevini

\begin{abstract}
*Department of Neuroscience, NOCSAE Hospital, University of Modena e Reggio Emilia, Modena, Italy; $†$ Neurology Unit, Epilepsy Centre, San Paolo Hospital, Milan, Italy; $\ddagger$ Department of Neuroscience, University of Parma, Parma, Italy; and
\end{abstract} \\ $\S$ Department of Medicine, Surgery, and Dentistry, Faculty of Medicine and Surgery, University of Milan, Milan, Italy
}

\section{SUMMARY}

Studies in animal models and patients with epilepsy have suggested that basal ganglia circuits may control epileptic seizures and that striatal dopaminergic transmission may play a role in seizure modulation and interruption. Chromosome $20[\mathrm{r}(20)]$ syndrome is a well-defined chromosomal disorder characterized by epilepsy, mildto-moderate mental retardation, and lack of recognizable dysmorphic features. Epilepsy is often the most important clinical manifestation of the syndrome, with prolonged episodes of nonconvulsive status epilepticus suggesting dysfunction in the seizure control system. We present the ictal blood oxygen level-dependent (BOLD) changes in brief seizures recorded by means of electroencephalography-functional magnetic resonance imaging (EEG-fMRI) coregistration in a patient with $[\mathrm{r}(20)]$ syndrome. We observed ictal BOLD increments in a cortical-subcortical network involving substantia nigrastriatum and frontal cortex. At present, this is the first functional neuroimaging evidence of the involvement of the nigrostriatal system during ictal EEG discharges in $[r(20)]$ syndrome supporting a role of the basal ganglia circuits in human epileptic seizures.

KEY WORDS: Ring chromosome 20 epilepsy, EEG-fMRI, BOLD, Basal ganglia, Substantia nigra.
Studies in animal models and patients with epilepsy have suggested that basal ganglia circuits may control epileptic seizures and that striatal dopaminergic transmission may play a key role in seizure modulation and interruption (Gale, 1992). Chromosome 20 [r(20)] syndrome is a well-defined chromosomal disorder characterized by epilepsy, mild-tomoderate mental retardation, and lack of recognizable dysmorphic features (Inoue et al., 1997; Canevini et al., 1998). Epilepsy is often the most important clinical manifestation of the syndrome, with prolonged episodes of nonconvulsive status epilepticus (NCSE) suggesting dysfunction in the seizure control system. In this line, recent interictal positron emission tomography (PET) and single photon emission computed tomography (SPECT) studies, suggested that dopaminergic dysfunction in the nigrostriatal system could have a role in the genesis and maintenance of seizures in [r(20)] syndrome (Biraben et al., 2004; Bouilleret et al., 2005).

Besides NCSE, Augustijn et al. (2001) and Vignoli et al. (2009) reported the occurrence of subtle, nocturnal frontal

Accepted May 10, 2012; Early View publication June 27, 2012.

Address correspondence to Stefano Meletti, Via Giardini, 1355, Nuovo Ospedale Civile, Modena, Italy. E-mail: stefano.meletti@unimore.it

Wiley Periodicals, Inc.

(C) 2012 International League Against Epilepsy lobe seizures as a characteristic ictal pattern of this syndrome. These seizures are characterized by a burst of diffuse but frontally predominant high-voltage fast activity clinically associated with minimal to moderate motor activity.

We present the ictal blood oxygen level-dependent (BOLD) changes in brief seizures recorded by means of electroencephalography-functional magnetic resonance imaging (EEG-fMRI) coregistration in a patient with [r(20)] syndrome. We observed ictal BOLD increments in a cortical-subcortical network involving substantia nigra-striatum and frontal cortex. At present, this is the first description of ictal basal ganglia involvement in this syndrome.

\section{Methods}

A 14-year-old girl was investigated by means of simultaneous EEG-fMRI coregistration in the context of an ongoing project on $[\mathrm{r}(20)]$ syndrome. Approval was granted by the human ethic committee of the University of Modena and Reggio Emilia, Italy. Written informed consent was obtained from her parents. The patient's karyotype was mosaic 46, XX, r(20)[53]/46, XX[47]. Epilepsy in the form of epileptic encephalopathy started at 9 years (Vignoli et al., 2009). At the present time, she has daily brief seizures 
during drowsiness and sleep characterized by minimal motor behavior (eye-opening with eyelid flutter) or sudden fear, followed by simple motor automatisms. Ictal EEG was characterized by a high-amplitude slow wave followed by a brief discharge of fast polyspikes predominant over the frontal leads (Fig. 1A).

\section{EEG and fMRI acquisition}

Scalp EEG has been recorded by means of a 32-channel MRI-compatible EEG recording system (Micromed, Mogliano Veneto, Italy). Electrodes were placed according to conventional 10-20 locations. Prior to in-magnet EEG recording, 30 min out-of magnet EEG was collected in a
Figure I.

(A) Example of one seizure recorded during routine video-EEG monitoring. The event is characterized by a diffuse discharge of fast spikes followed by high amplitudes slow waves. The EEG was recorded by 19 electrodes according to the 10-20 system and represented in monopolar reference. (B) Example of one seizure recorded during the EEGfMRI session. EEG was recorded by 32 electrodes and represented in monopolar reference. The ictal pattern is similar to that recorded in A. This kind of EEG quality was obtained after gradient-artifact removing and after correction of the cardio-ballistogram artifact, as described in the Method section. Epilepsia (c) ILAE

\section{A VIDEO-EEG RECORDING}

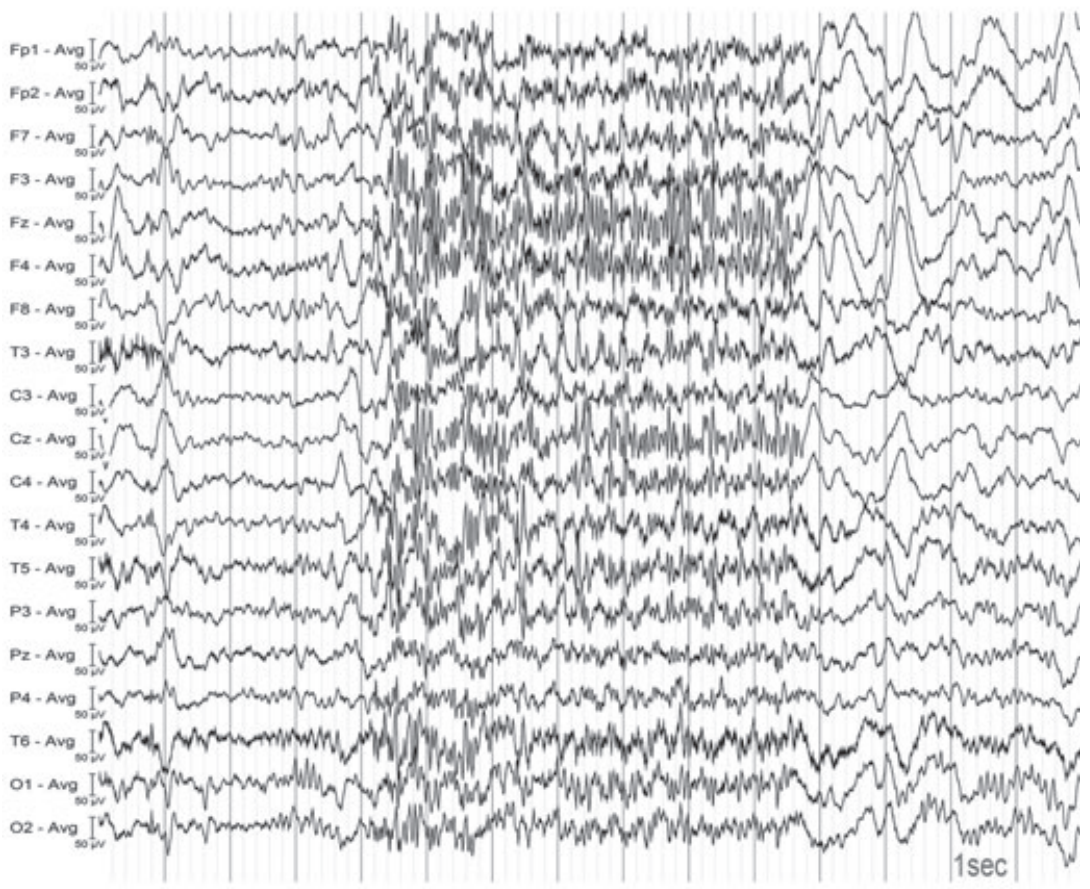

B EEG-fMRI RECORDING

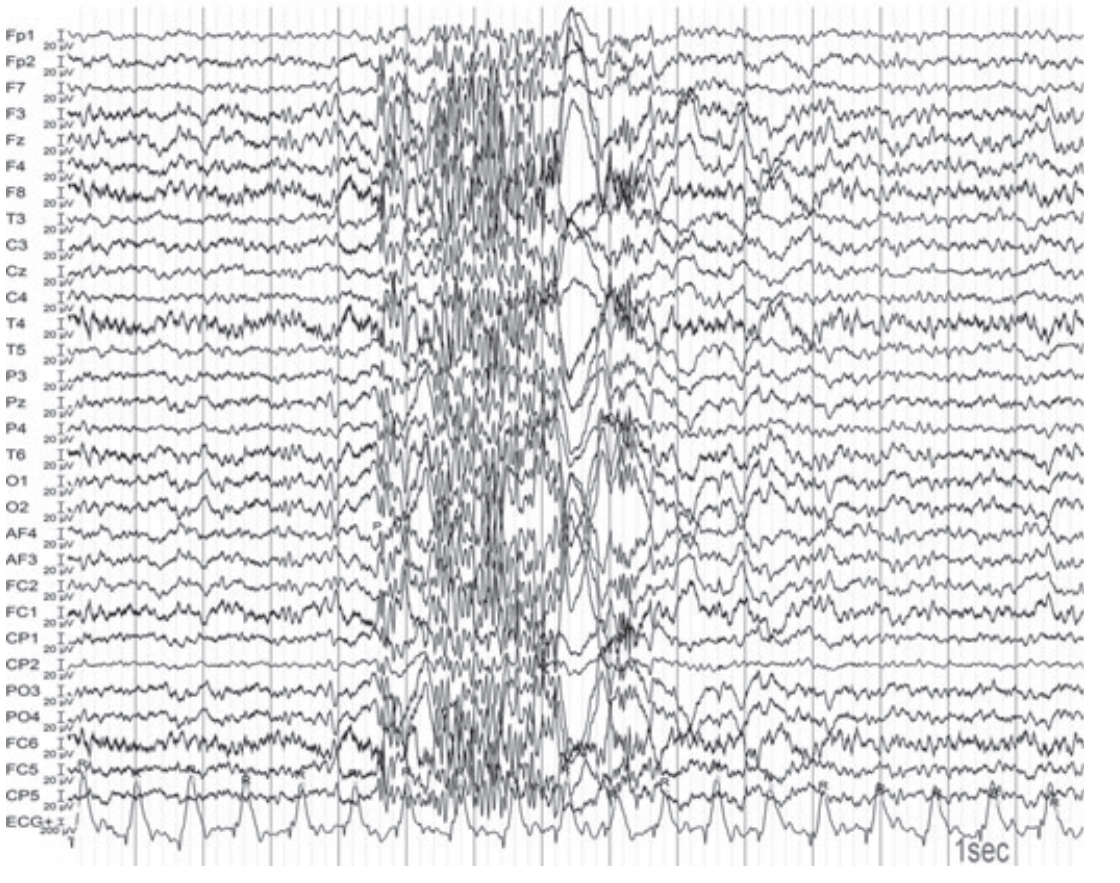


room adjacent to the scanner. Foam pads were used to help secure the EEG leads, minimize motion, and improve patient comfort. Data were transmitted via an optic fiber cable from the amplifier (1,024 Hz sampling rate) to a computer located outside the scanner room. To avoid saturation, the EEG amplifiers have a resolution of 22 bits with a range of $\pm 25.6 \mathrm{mV}$.

The patient's behavior was constantly observed and recorded by means of a small camcorder positioned on the head coil inside the scanner pointing to the patient's face to obtain a split-screen video-EEG documentation during the fMRI recording.

Functional data have been acquired using a Philips Intera system at 3T and a gradient-echo echo-planar sequence from 30 axial contiguous slices (TR $=3,000 \mathrm{msec}$; in-plane matrix $=64 \times 64$; voxel size: $4 \times 4 \times 4$ ) over three 10 -min sessions (200 volumes/session) with continuous simultaneous EEG recording. A high-resolution $\mathrm{T}_{1}$-weighted anatomic image has been acquired to allow accurate anatomic localization of activations/deactivations. The volume consisted of 170 sagittal slices ( $\mathrm{TR}=9.9 \mathrm{msec}$; $\mathrm{TE}=4.6 \mathrm{msec} ;$ in plane matrix $=256 \times 256$; voxel size $=$ $1 \times 1 \times 1 \mathrm{~mm})$.

\section{EEG data analysis}

BrainQuick System Plus software (Micromed) was used for offline correction of the gradient artifacts (Allen et al., 2000) and filtering of the EEG signal.

In addition, the EEG data were exported in the .edf format and reviewed and analyzed by means of the BrainVision Analyzer 2.0 software (Brain Products, Munich, Germany). To remove the ballistocardiographic artifact from the recording, an independent component analysis (ICA) was performed on dataset. Each component was subsequently segmented around electrocardiography (EKG) R-peaks, and components responsible of the $80 \%$ of the variance were removed (Debener et al., 2009). The in magnet video-EEG recordings were then reviewed in a referential montage by two experienced electroencephalographers and compared to the subject's EEG recording outside the scanner in order to mark EEG events with the characteristics of discharges of high-voltage fast spikes.

For subsequent time-frequency analysis of selected EEG events, data were filtered using a 1-250 band-pass II order filter, and then segmented around each marker so as to investigate the time window from $-2 \mathrm{~s}$ up to $8 \mathrm{~s}$ with respect to the seizure onset. A complex Morlet wavelet was performed to obtain a time-frequency description of these events, investigating 15 frequency bands ranging from $1-60 \mathrm{~Hz}$.

\section{fMRI data analysis}

The Matlab 7.1 and SPM5 (Wellcome Department of Imaging Neuroscience, London, United Kingdom) software was used for data analysis. All functional volumes were slice time corrected, realigned to the first volume acquired, normalized using the standard Montreal Neurological Institute (MNI) template as implemented din SPM5 and smoothed with a $12 \times 12 \times 12 \mathrm{~mm}$ full-width half maximum (FWHM) Gaussian kernel. Data analysis was performed by means of the SPM5 general linear model (GLM) using EEG events of interest as regressors convolved with the standard hemodynamic response function (HRF). EEG events were represented as variable-duration events beginning at the onset of the first spike and ending upon the termination of the high-voltage fast activity. The six motion parameters derived from the fMRI preprocessing (translation and rotation in the $\mathrm{X}, \mathrm{Y}$, and $\mathrm{Z}$ direction, respectively) were used as covariates in the GLM. Movement artifacts individuated by the analysis of EEG and video-recordings (eye-blink, deglutition, head movements) were considered as confounders in the model.

One-tailed $t$-tests were applied to test for regional BOLD increases or decreases in relationship with the EEG events of interest. At the voxel level, the significance level was set at $\mathrm{p}<0.05$ after correction for multiple comparisons across the whole brain using the family wise error (FWE) correction method as implemented in SPM5 (Friston et al., 1995). This corresponds to $t$-values above 4.7. An extent threshold of five contiguous voxels was also applied. Results were superimposed onto the individual high-resolution $\mathrm{T}_{1}$-weighted images normalized into the MNI space.

Coordinates in Talairach space were obtained by applying the Matthew Brett correction (mni2tal: http://www.mrccbu.cam.ac.uk/Imaging/mnispace.html) to the SPM-MNI coordinates.

\section{Results}

Thirty-one brief seizures were recorded during the EEGfMRI acquisitions with a mean duration of $3 \mathrm{~s}$ (one example is depicted in Fig. 1B). The seizures were characterized by eye-opening with eyelid flutter.

In Fig. 2A,B is reported the average of the timefrequency analysis for the 31 examined discharges of fast spikes. The events were characterized by a maximum band power between 20 and $40 \mathrm{~Hz}$.

The analysis of fMRI signal changes showed marked BOLD increases in the midbrain (local maxima right substantia nigra: $\mathrm{x}=8 ; \mathrm{z}=-10 ; \mathrm{y}=-17$ ), head of the caudatus, visual, opercular-insular, sensory-motor, and medial prefrontal cortex bilaterally (Fig. 2C). No BOLD signal decrements were detected.

\section{Discussion}

This is the first functional neuroimaging evidence of the involvement of the nigrostriatal system during ictal EEG discharges in $[\mathrm{r}(20)]$ syndrome, supporting a role of the basal ganglia circuits in human epileptic seizures. 
Figure 2.

Ictal BOLD changes during fast discharges. (A) Example of one ictal event as recorded from the frontal leads (F3, Fz, and F4) that has been used for the SPM analysis. (B) Timefrequency plots for $\mathrm{F} 3, \mathrm{Fz}$, and F4 (average of the $3 \mathrm{I}$ events that have been recorded). The plots represent the average power for the different frequency bands over time from I s before to $4 \mathrm{~s}$ after seizure start (time 0). Note that the ictal discharges were characterized by a mean frequency about $20-40 \mathrm{~Hz}$ (red color). (C) On the left, a sagittal brain section $(x=8)$ shows midbrain, caudate, visual, and medial prefrontal cortex increments of BOLD signal related to fast discharges. On the right, three axial images corresponding to the plane of the blue lines are reported. (I) Axial image $(z=-10)$ showing BOLD increase in the midbrain; (2) axial image at the level of caudate nuclei $(z=2)$. In both I and 2 , BOLD increments are evident also at frontoopercular and visual cortical areas bilaterally. (3) Axial image $(z=50)$ showing activations in the medial frontal lobe and sensory-motor cortex bilaterally. Activated cluster were superimposed on the patient's $T_{\text {I }}$ image ( $p<0.05$ corrected for multiple comparisons FWE; $k>5$ voxels).

Epilepsia (c) ILAE

\section{A SINGLE EVENT}

$$
\mathrm{F}_{3}
$$

$\mathrm{F}_{3}$
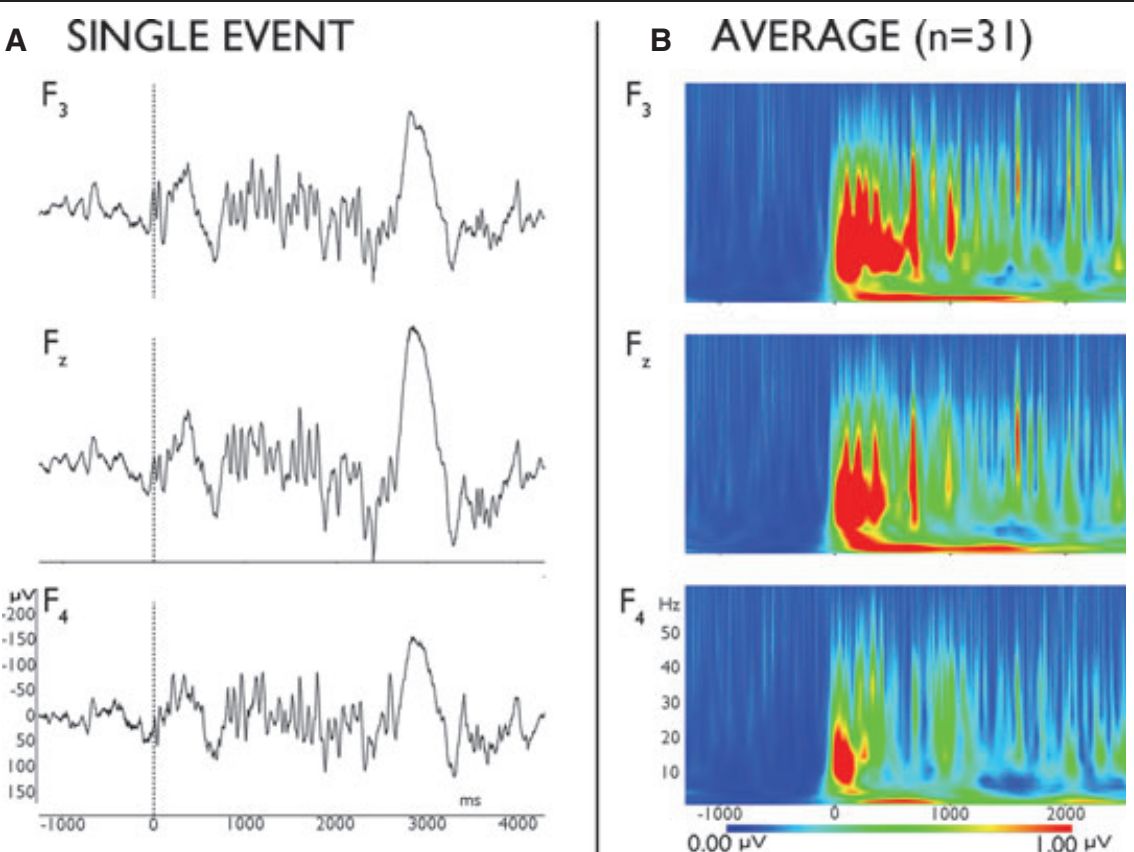

$\mathrm{F}_{\mathrm{z}}$
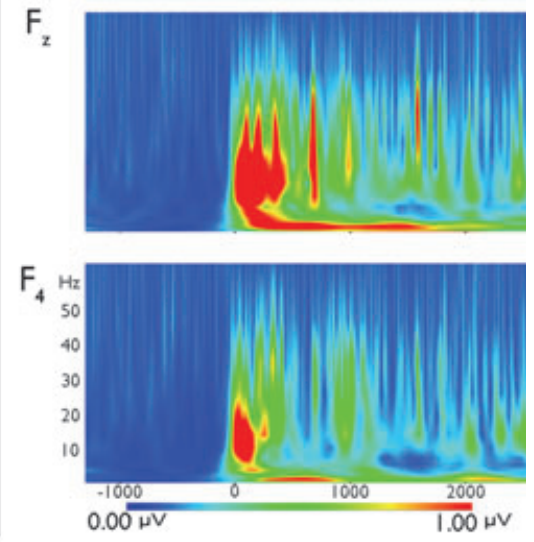

\section{c BOLD RESULTS}

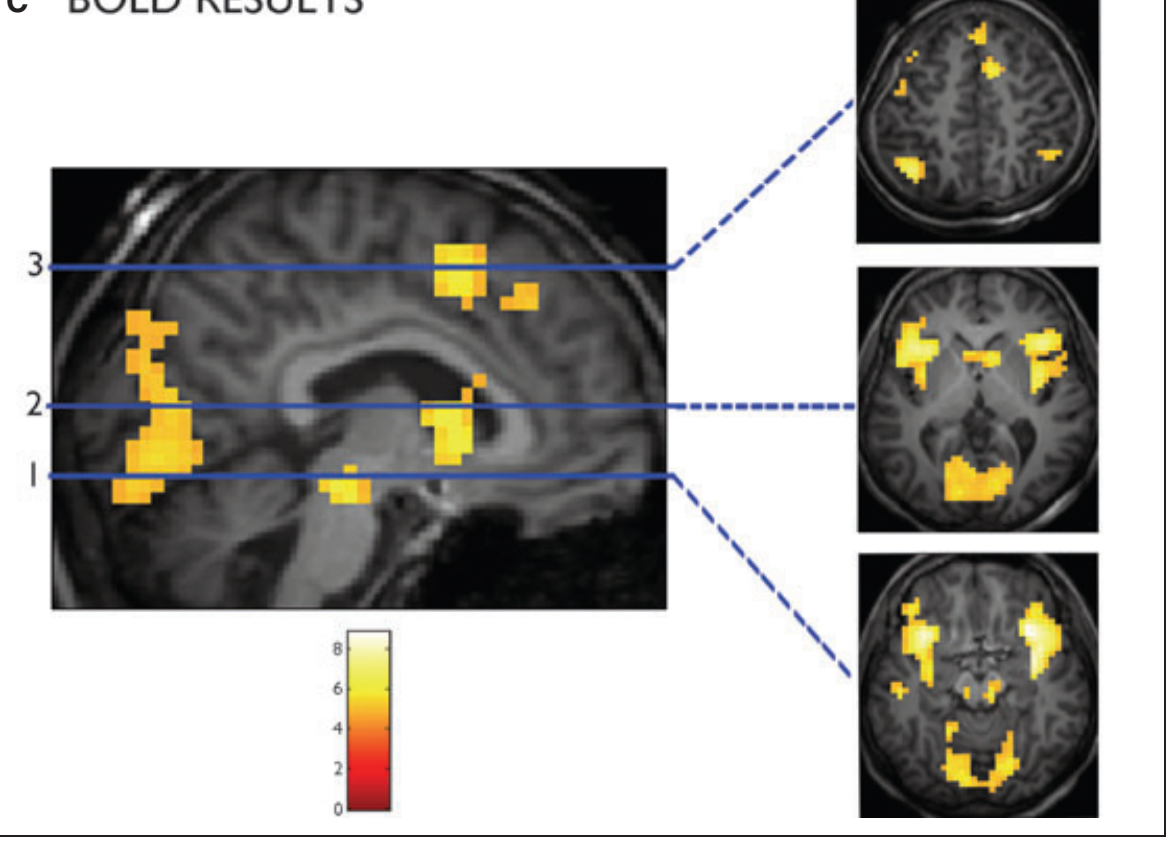

Obviously, the present evidence is limited to a particular seizure types (brief high-voltage fast activity) in a single patient with a rare chromosomal disorder. However, we believe that this observation is important to prompt further studies to assess the role of the nigrostriatal system in this disorder as well as in other human drug-resistant epilepsies. In this line, our findings expand previous results obtained by PET studies documenting an interictal decrease of L-DOPA uptake in the striatum and substantia nigra in patients with $[\mathrm{r}(20)]$ syndrome and in patients with other drug-resistant epilepsies (Biraben et al., 2004; Bouilleret et al., 2005). Overall these findings support future investigations of different therapeutic strategies in $[\mathrm{r}(20)]$ syndrome, both pharmacologic (dopaminergic) or nonpharmacologic (deep brain stimulation) to target the activity of basal ganglia circuits.

In addition to ictal midbrain-caudate increments of BOLD signal, we observed activations of cortical regions involving the frontal lobe. In particular, the activations involved the frontoopercular cortex bilaterally and the 
medial frontal cortex. These ictal BOLD increments are concordant with the hypothesis that seizures in ring-20 epilepsy reflect frontal lobe circuitry dysfunction. This idea is supported by the clinical semiology of subtle nocturnal seizures (Augustijn et al., 2001; Vignoli et al., 2009), by recent ictal magnetoencephalography documentation of medial frontal lobe involvement (Tanaka et al., 2004), and by the genetic evidence of frontal lobe involvement in epilepsy caused by mutations within the distal long arm of chromosome 20 (autosomal dominant frontal lobe epilepsy).

Finally, we observed BOLD increments in the visual areas and in the sensory-motor cortex bilaterally. At present we do not have a hypothesis to explain these BOLD signal changes, and future studies including more patients with [r(20)] syndrome should be performed.

In conclusion, EEG-fMRI is a valuable technique that can provide new information to elucidate cortical-subcortical networks in rare encephalopathies revealing the participation of brainstem structures in the epileptogenic network (Siniatchkin et al., 2011).

\section{ACKNOWLedgments}

This work was supported by a grant of "Fondazione Cassa di Risparmio di Modena,” Modena, Italy: Ricerca Internazionale 2010.

\section{Disclosure}

None of the authors has any conflict of interest to disclose. We confirm that we have read the Journal's position on issues involved in ethical publication and affirm that this report is consistent with those guidelines.

\section{REFERENCES}

Allen PJ, Josephs O, Turner R. (2000) A method for removing imaging artifact from continuous EEG recorded during functional MRI. Neuroimage 12:230-239.

Augustijn PB, Parra J, Wouters CH, Joosten P, Lindhout D, van Emde Boas W. (2001) Ring chromosome 20 epilepsy syndrome in children: electroclinical features. Neurology 57:1108-1111.

Biraben A, Semah F, Ribeiro MJ, Douaud G, Remy P, Depaulis A. (2004) PET evidence for a role of the basal ganglia in patients with ring chromosome 20 epilepsy. Neurology 63:73-77.

Bouilleret V, Semah F, Biraben A, Taussig D, Chassoux F, Syrota A, Ribeiro MJ. (2005) Involvement of the basal ganglia in refractory epilepsy: an 18F-fluoro-1-DOPA PET study using 2 methods of analysis. J Nucl Med 46:540-547.

Canevini MP, Sgro V, Zuffardi O, Canger R, Carrozzo R, Rossi E, Ledbetter D, Minicucci F, Vignoli A, Piazzini A, Guidolin L, Saltarelli A, Dalla Bernardina B. (1998) Chromosome 20 ring: a chromosomal disorder associated with a particular electroclinical pattern. Epilepsia 39:942-951.

Debener S, Kranczioch C, Gutberlet I. (2009) Origin and removal of the ballistocardiogram artefact. In Mulert C, Lemieux L (Eds) EEG-fMRI: physiology, technique and applications. Springer, Berlin, pp. 135-151.

Friston KJ, Holmes AP, Worsley KP, Poline JB, Frith C, Frackowiak RSJ. (1995) Statistical parametric maps in functional imaging: a general linear approach. Hum Brain Mapp 2:189-210.

Gale K. (1992) Subcortical structures and pathways involved in convulsive seizure generation. J Clin Neurophysiol 9:264-277.

Inoue Y, Fujiwara T, Matsuda K, Kubota H, Tanaka M, Yagi K, Yamamori K, Takahashi Y. (1997) Ring chromosome 20 and nonconvulsive status epilepticus. A new epileptic syndrome. Brain 120:939-953.

Siniatchkin M, Coropceanu D, Moeller F, Boor R, Stephani U. (2011) EEG-fMRI reveals activation of brainstem and thalamus in patients with Lennox-Gastaut syndrome. Epilepsia 52:766-774.

Tanaka N, Kamada K, Takeuchi F. (2004) Ictal magnetoencephalographic study in a patient with ring 20 syndrome. J Neurol Neurosurg Psychiatry 75:488-490.

Vignoli A, Canevini MP, Darra F, La Selva L, Fiorini E, Piazzini A, Lazzarotto F, Zucca C, Dalla Bernardina B. (2009) Ring chromosome 20 syndrome: a link between epilepsy onset and neuropsychological impairment in three children. Epilepsia 50:2420-2427. 